\title{
Strategies to simplify complex medication regimens
}

J Simon Bell, Brigid Mclnerney Esa YH Chen, Phillip J Bergen, Lorenna Reynolds, Janet K Sluggett

\section{Background \\ Older people use increasingly complex medication regimens. Complex regimens are challenging to administer, particularly for those with cognitive impairment, frailty, poor eyesight or limited dexterity. Complex regimens have been linked to non-adherence, medication errors and hospital admissions.}

\section{Objective}

The aim of this article is to describe strategies to reduce the complexity of medication regimens in community and residential aged care settings.

\section{Discussion}

Medication regimen simplification is the process of reducing medication burden through strategies such as consolidating dosing times, standardising routes of administration, using long-acting rather than shorter-acting formulations, and switching to combination products in place of single-ingredient products. Obtaining a best possible medication history, ensuring appropriateness of current therapy, and deprescribing are important steps prior to implementing regimen simplification. Implementing such strategies should be based on a discussion and consideration of patient preferences, and include clinical judgement to limit the risk of unintended consequences for patients or carers.
MEDICATION MANAGEMENT is a component of treatment burden experienced by people with multimorbidity. ${ }^{1}$ Burden can arise as a result of needing to access, administer and monitor medications, and through experiencing adverse medication events. This burden is exacerbated by the use of complex medication regimens. Two-thirds of Australians aged $\geq 75$ years use five or more regular medications. ${ }^{2}$ More than half of residents of Australian residential aged care facilities (RACFs) use nine or more regular medications, and one-third have five or more daily medication administration times. ${ }^{3}$ Complex medication regimens may be linked to the application of multiple disease-specific clinical practice guidelines in the context of multimorbidity, inertia arising from reluctance to modify treatment initiated by another prescriber, and frequent transitions of care. ${ }^{4}$ Medication regimen simplification is consistent with the concept of minimally disruptive medicine, which refers to minimising the treatment burden that arises from asking patients to adhere to multiple treatments, guidelines and recommendations. ${ }^{1}$

\section{What makes a medication regimen complex?}

Complexity of a medication regimen is correlated with the number of prescription, non-prescription and complementary and alternative medications (CAMs). ${ }^{3}$ Complexity can also arise because of the number of daily medication administration times, multiple or complicated dose forms, and special administration instructions (eg crush tablets, take with food). ${ }^{3}$

Clinicians may underestimate the difficulty people experience interpreting dosing instructions. This can result in variability in medication self-administration practices. ${ }^{5,6}$ A US study showed that when patients aged 55-74 years were presented with the same hypothetical seven-medication regimen suitable for four times daily dosing, patients took medications on three to 14 different occasions throughout the day. ${ }^{6}$ Patients may unnecessarily adjust their daily routine to accommodate restrictive dosing instructions. Lindquist et al reported that older people may delay leaving the house or going to bed to comply with specific dosing instructions. ${ }^{5}$ To understand the true complexity of a medication regimen, it is necessary to actively and non-judgementally ask about use of prescription medications, non-prescription medications and CAMs, the latter often not routinely disclosed in consultations. ${ }^{7}$ This should include a discussion of both regular and as-needed (PRN) medication use.

\section{Why reduce medication regimen complexity?}

Complex medication regimens are associated with a higher number of errors (eg self-administration errors) and an increased risk of hospitalisation. ${ }^{8}$ Medication regimen complexity is also 
an independent predictor of hospital discharge to non-home settings. ${ }^{9}$ There is emerging although inconsistent evidence that the overall complexity of an older person's medication regimen is associated with non-adherence, ${ }^{8}$ with once-daily dosing associated with better adherence than twice, three times or four times daily dosing. ${ }^{10}$ A review of seven studies reported less frequent dosing is associated with similar or higher health-related quality of life in people with angina, asthma, chronic obstructive pulmonary disease, Parkinson's disease and seizures. ${ }^{11}$

Overall complexity should be considered in the context of an individual's capacity to self-manage their medication regimen. Paradoxically, the most complex regimens are often prescribed to those with the least capacity to manage. ${ }^{3}$ This may include people with cognitive impairment, who are frail, have poor eyesight or limited dexterity. ${ }^{3,12}$ For example, older people with Parkinson's disease or rheumatoid arthritis may have difficulty opening child-resistant containers and using medical devices (eg inhalers) because of dexterity changes. Poor health literacy may be associated with an inability to read or interpret complex instructions for medication use, leading to an increased risk of medication errors. When presented with the same medications, people with lower health literacy report taking their medications on more occasions throughout the day than those with higher health literacy. ${ }^{6}$

While all older people may benefit from reduced treatment burden, other potential benefits are setting dependent. For community-dwelling older people, capacity to self-manage a medication regimen may be a requirement for continuing to live independently at home. Reducing the number of daily administration times to coincide with home visits by nurses, carers or family members may improve medication adherence. In residential aged care, simplifying complex medication regimens may assist residents with dementia-related swallowing difficulties and minimise nursing time required to prepare and administer medications. This may allow more time for nurses to focus on safe administration practices or other direct care activities. Medication administration is time-consuming and challenging for informal carers, nurses and personal care workers. ${ }^{13,14}$

\section{How can medication regimen complexity be reduced?}

Steps for simplifying a complex medication include obtaining a best possible medication history (BPMH), ensuring appropriateness of current therapy and deprescribing, and medication regimen simplification. It is necessary to have an accurate medication list before initiating the process of deprescribing and medication regimen simplification. ${ }^{15-17}$ Deprescribing and medication regimen simplification are complementary services. Deprescribing involves the 'stepwise reduction of unnecessary or potentially inappropriate medications after consideration of therapeutic goals, benefits and risks, and medical ethics'. ${ }^{17}$ Medication regimen simplification refers to reducing medication burden through strategies such as consolidating dose times and routes of administration. ${ }^{16}$ Unlike deprescribing, medication regimen simplification on its own does not seek to change the therapeutic intent of the regimen.

\section{Obtain a best possible medication history}

A BPMH is a current and complete list of an individual's prescription, non-prescription and CAMs compiled from various sources including the patient or carer. ${ }^{18} \mathrm{~A}$ BPMH should include details such as medication dose, frequency and route of administration, recognising that these may not be in accordance with the prescriber's intent. Any discrepancies between the BPMH and the intended medication regimen should be clarified and documented. ${ }^{18}$ Medication reconciliation is recommended at various points during patient care, and is a starting point for deprescribing and regimen simplification. ${ }^{19}$

\section{Ensuring appropriateness of current therapy and deprescribing}

Once a BPMH has been obtained, it is important to confirm all medications are clinically indicated and appropriate. Home Medicine Reviews (HMRs) and Residential Medication Management Reviews (RMMRs) are collaborative services provided by general practitioners and pharmacists that involve identification of possible medication-related problems. ${ }^{19-21}$ For this reason HMRs and RMMRs may be useful for identifying opportunities for deprescribing. Pooled analyses of seven RMMR studies indicated $16 \%$ of pharmacist recommendations related to medication cessation. ${ }^{20}$ While overall evidence for medication review is mixed, HMR and RMMR in Australia has been associated with reduced numbers of medications, decline in potentially inappropriate medication use and fewer hospitalisations..$^{20,21}$ Deprescribing is underpinned by complex decision-making processes, ${ }^{22}$ and requires shared decision making with prescribers, patients and their family, pharmacists, nurses and allied health professionals. ${ }^{19}$ Use of a decision aid in relation to deprescribing proton-pump inhibitors has been trialled with mixed success in Canada. ${ }^{23}$ However, the wider body of literature on decision aids suggests these may be valuable to promote active involvement in deprescribing decisions by helping patients become more knowledgeable, better informed and clearer about their values. ${ }^{24}$ It is important for clinicians to initiate discussions about deprescribing, because willingness to deprescribe is higher if physicians reassure patients it is possible. ${ }^{25}$

\section{Medication regimen simplification}

When all current medications are deemed clinically appropriate, it may be possible to further reduce a patient's medication burden through medication regimen simplification strategies (Box 1).

When simplifying a regimen, the clinical benefit of administering medications at a specific time of day (eg administering a statin at night, thyroxine before breakfast) may need to be balanced against the likely 
benefits achieved through reducing the overall regimen complexity. The decision to reduce complexity should always involve a discussion with the patient and their carer to elucidate their preferences and perspectives.

The Medication Regimen Simplification Guide for Residential Aged CarE (MRS GRACE) is a newly developed, implicit five-step process for guiding the simplification process (Box 2). ${ }^{16} \mathrm{Using}$ the MRS GRACE tool, two pharmacists independently simplified 29 and 30 out of 50 residents' medication regimens, respectively, with fair agreement. ${ }^{16}$ Simplification was possible for the majority of residents with four or five daily medication administration times. A cluster randomised controlled trial in South Australian RACFs showed that applying the principles in the MRS GRACE tool reduced residents' number of daily administration times (odds ratio -0.36 ; 95\% confidence interval: $-0.63,-0.09$;

\section{Box 1. Medication simplification strategies ${ }^{15,16}$}

Regimens can be simplified through strategies such as:

- administering medications at the same time

- using long-acting rather than shorteracting formulations

- using combination products rather than multiple single-ingredient products

- consolidating routes of administration.
$P=0.01$ ) at the four-month follow-up, with no measurable impact on secondary outcomes including resident satisfaction, quality of life, falls, hospitalisations or mortality. ${ }^{15}$ However, the impact of the one-off simplification intervention on number of daily administration times was sustained over 12 months. ${ }^{26}$ A multi-component intervention that involved obtaining a BPMH and medication regimen simplification was successfully piloted among 25 recipients of community-based home care services, with simplification opportunities identified for 14 individuals. ${ }^{27}$

Box 3 provides an example of how a hypothetical medication regimen may be simplified. This example incorporates the acquisition of a BPMH, ensuring clinical appropriateness and deprescribing, and medication regimen simplification.

\section{Reasons for not simplifying medication regimens}

Clinical judgement is required before implementing simplification strategies to avoid any unintended consequences or inadvertently reduce medication adherence. There are circumstances in which simplification may not be appropriate. ${ }^{16}$ These include patientrelated factors (eg patient prefers to spread medication-taking across day, patient is unable to swallow modifiedrelease formulations whole), likelihood of medication interactions (eg orthostatic hypotension from co-administration of

\section{Box 2. Medication Regimen Simplification Guide for Residential Aged CarE (MRS GRACE) $^{16}$}

Consideration can be given to administering all medications at the same time each day unless the following apply:

1. Is there a resident-related factor that precludes simplification?

2. Is there a regulatory or safety imperative that precludes simplification?

3. Is simplification likely to result in any clinically significant drug-drug, drug-food, or drug-time interactions?

4. Is there no alternative formulation available that can support less complex dosing?

5. Is simplification likely to result in any unintended consequences for the resident or facility?

Reproduced from Chen EYH, Sluggett JK, Ilomäki J, et al, Development and validation of the Medication Regimen Simplification Guide for Residential Aged CarE (MRS GRACE), Clin Interv Aging 2018;13:975-86, doi: 10.2147/CIA.S158417. A full explanatory statement for each simplification criterion is available in Chen et al.16

cardiovascular medications), possible unintended consequences (eg need for invasive monitoring) and setting-specific factors (eg qualified staff not available to administer medications at specific times).

\section{Medication management strategies}

Dose administration aids (DAAs) supplied by pharmacies can be used to aid medication management in community and RACF settings. Examples of DAAs include compartmentalised plastic boxes (eg Dosette), blister packs (eg MedicoPak, Webster-Pak) and sachet systems (eg APHS medication sachets, MPS Packettes). DAAs are widely used to aid medication administration in RACFs. ${ }^{19}$ Potential benefits of DAA use in the community include increased communication between healthcare professionals and better disease control. ${ }^{28}$ However, DAAs do not address intentional non-adherence. DAAs are most effective in patients motivated and willing to take their medications, and who have the ability to use the DAA. ${ }^{28}$ A DAA may not be appropriate for all patients for example, if the regimen is frequently changing, or the medications are not appropriate for inclusion in a DAA (eg inhalers, hygroscopic tablets) ${ }^{28}$ Reducing the complexity of a patient's medication regimen may also avoid the need for a DAA. Resources in relation to medication management strategies are provided at the end of this article.

\section{Activities post-regimen simplification}

Medication regimen simplification includes documenting and communicating regimen changes with the patient and others involved in medication management (eg medical specialists, RACF staff, community pharmacists, carers, nurses, providers of communitybased home care services). Ongoing assessment of the suitability of the new regimen and monitoring for any potential unintended consequences is also important. The provision and maintenance of an up-to-date medication list may aid the patient's understanding 


\section{Box 3. Example simplification of a hypothetical medication regimen}

\section{Obtain a best possible medication history}

Mrs AB is a patient at your general practice clinic. You create a best possible medication history through non-judgementally interviewing Mrs AB about her current medications, proactively asking about any non-prescription medications or complementary and alternative medications (CAMs), reviewing medical records and inspecting her dose administration aid.

\begin{tabular}{lcccc}
\hline & \multicolumn{3}{c}{ Dose } \\
\cline { 2 - 4 } Medication & Breakfast & Lunch & Dinner & Bed \\
\hline Atorvastatin $10 \mathrm{mg}$ & & & 1 \\
\hline Amlodipine $5 \mathrm{mg}$ & 1 & 2 & 2 \\
\hline Metformin $500 \mathrm{mg}$ & 1 & 2 & \\
\hline Paracetamol $500 \mathrm{mg}$ & 2 & & \\
\hline Pantoprazole $40 \mathrm{mg}$ & 1 & & 1 \\
\hline Ferrous sulfate $325 \mathrm{mg}$ CR & 1 & & 1 \\
\hline Latanoprost 0.005\% eye drops & & & \\
\hline Timolol 0.5\% eye drops & & & \\
\hline
\end{tabular}

\section{Ensure appropriateness of current therapy and deprescribing}

You review the ongoing clinical appropriateness of each medication and discuss deprescribing with Mrs AB. With permission you also involve Mrs AB's carer.

- Mrs AB's GORD symptoms are well controlled. You suggest reducing the dose of pantoprazole to $20 \mathrm{mg}$ daily with the view to possible cessation.

- Recent iron levels suggest no sign of iron deficiency. On interview, Mrs AB discloses that she started taking iron tablets because her neighbour suggested they would 'make her feel more energetic'. You suggest cessation.

- Mrs AB states that her chronic pain has improved since commencing an exercise regimen recommended by her physiotherapist. You suggest reducing the daily paracetamol dose, with a plan to review and adjust as appropriate.

\begin{tabular}{|c|c|c|c|c|}
\hline \multirow[b]{2}{*}{ Medication } & \multicolumn{4}{|c|}{ Dose } \\
\hline & Breakfast & Lunch & Dinner & Bed \\
\hline Atorvastatin $10 \mathrm{mg}$ & & & & 1 \\
\hline Amlodipine 5 mg & 1 & & & \\
\hline Metformin 500 mg & 1 & & 1 & \\
\hline Paracetamol $500 \mathrm{mg}$ & 2 & 2 & & 2 \\
\hline Pantoprazole 20 mg & 1 & & & \\
\hline Latanoprost $0.005 \%$ eye drops & & & & 1 \\
\hline Timolol $0.5 \%$ eye drops & & & & \\
\hline
\end{tabular}

Box continues on page 47. and use of the new regimen, and facilitate the communication of the regimen to other health professionals and across transitions of care. ${ }^{29}$

\section{Additional resources for medication management}

\section{Medication management and living with multiple medications}

- NPS MedicineWise - Living with multiple medications: Patients and health professionals share their experiences, www.nps.org.au/livingwith-multiple-medicines

- The Royal Australian College of General Practitioners. Medication management. In: RACGP aged care clinical guide (Silver Book). 5th edn. East Melbourne, Vic: RACGP, 2019.

- Hilmer SN, Gnjidic D, Le Couteur DG. Thinking through the medication list: Appropriate prescribing and deprescribing in robust and frail older patients. Aust Fam Physician 2012;41(12):924-28.

\section{Best possible medication history and medication reconciliation}

- Australian Commission on Safety and Quality in Health Care (ACSQHC) Medical reconciliation: Resources for obtaining a best possible medication history, www.safetyandquality.gov. $\mathrm{au} /$ medical-reconciliation-resourcesobtaining-best-possible-medicationhistory

\section{Deprescribing}

- The Royal Australian College of General Practitioners. Deprescribing. In: RACGP aged care clinical guide (Silver Book). 5th edn. East Melbourne, Vic: RACGP, 2019.

- Monash University and Victorian Government Department of Health and Human Services - Talking about deprescribing [video], https://vimeo. com/285078644/85ac97d038

- Potter K, Page A, Clifford K, EthertonBeer C. Deprescribing: A guide for medication reviews. J Pharm Pract Res 2016;46(4):358-67. doi: 10.1002/ jppr.1298. 


\section{Box 3. Example simplification of a hypothetical medication regimen (cont'd)}

\section{Medication regimen simplification}

Once you are satisfied all medications and doses are appropriate, you implement regimen simplification strategies with no changes to the therapeutic intent:

- atorvastatin prescribed in combination with amlodipine

- metformin switched to the XR formulation

- paracetamol rationalised to the CR formulation

- latanoprost prescribed in combination with timolol.

\begin{tabular}{|c|c|c|c|c|}
\hline \multirow[b]{2}{*}{ Medication } & \multicolumn{4}{|c|}{ Dose } \\
\hline & Breakfast & Lunch & Dinner & Bed \\
\hline Atorvastatin 10 mg/amlodipine 5 mg & 1 & & & \\
\hline Metformin XR $1000 \mathrm{mg}$ & 1 & & & \\
\hline Paracetamol 665 mg CR & 2 & & & 2 \\
\hline Pantoprazole $20 \mathrm{mg}$ & 1 & & & \\
\hline Latanoprost $0.005 \% /$ timolol $0.5 \%$ eye drops & & & & 1 \\
\hline
\end{tabular}

\section{Overall result}

- Daily administration times reduced from four to two

- Daily tablets reduced from 14 to seven

- Daily eye drop administrations reduced from two to one

- Eight medication products reduced to five

$C R$, controlled release; GORD, gastro-oesophageal reflux disease; $X R$, extended release

\section{Medication regimen simplification}

- Chen EYH, Sluggett JK, Ilomäki J, et al. Development and validation of the Medication Regimen Simplification Guide for Residential Aged CarE (MRS GRACE). Clin Interv Aging 2018;13:975-86. doi: 10.2147/CIA. S158417.

\section{Authors}

J Simon Bell BPharm (Hons), PhD, Professor and Director, Centre for Medicine Use and Safety, Faculty of Pharmacy and Pharmaceutical Sciences, Monash University, Vic.

Brigid Mclnerney BPharm, GradCertPharmPrac, MClinPharm, AACP, Senior Pharmacist, Community Care Services, Alfred Health, Vic

Esa YH Chen BPharm (Hons), Centre for Medicine Use and Safety, Faculty of Pharmacy and Pharmaceutical Sciences, Monash University, Vic Phillip J Bergen BSc, BPharm (Hons), GCAP, MTeach (Sec), PhD, Senior Research Officer, Microbiology, School of Biomedical Sciences, Monash University, Vic; Adjunct Research Fellow, Centre for Medicine Use and Safety, Faculty of Pharmacy and Pharmaceutical Sciences, Monash University, Vic
Lorenna Reynolds BPharm (Hons),

GradCertPharmPraclnt, Research Officer, Centre for Medicine Use and Safety, Faculty of Pharmacy and Pharmaceutical Sciences, Monash University, Vic Janet K Sluggett BPharm (Hons), GradDipClinEpid, PhD, FSHP, AACPA, Senior Research Fellow, UniSA Allied Health and Human Performance, University of South Australia, SA; Adjunct Research Fellow, Centre for Medicine Use and Safety, Monash University, Vic Competing interests: JSB reports grants from the National Health and Medical Research Council (NHMRC) Cognitive Decline Partnership Centre, the NHMRC Boosting Dementia Leadership Fellowship the Victorian Government Department of Health and Human Services, Dementia Australia Research Foundation, GlaxoSmithKline and several aged care provider organisations, outside the submitted work. EYHC is supported by postgraduate research scholarships from the NHMRC Cognitive Decline Partnership Centre and the Faculty of Pharmacy and Pharmaceutical Sciences (Monash University). $\mathrm{LR}$ is partly funded through the NHMRC Boosting Dementia Scheme. JKS is supported by an NHMRC Early Career Fellowship (grant number APP1156439).

Funding: The manuscript was prepared with funding support provided by the National Health and Medical Research Council (NHMRC) Cognitive Decline Partnership Centre.

Provenance and peer review: Not commissioned, externally peer reviewed.

Correspondence to:

Simon.Bell2@monash.edu

\section{References}

1. Trevena L. Minimally disruptive medicine for patients with complex multimorbidity. Aust J Gen Pract 2018;47(4):175-79. doi: 10.31128/AFP-10-174374.

2. Morgan TK, Williamson M, Pirotta M, Stewart K, Myers SP, Barnes J. A national census of medicines use: A 24-hour snapshot of Australians aged 50 years and older. Med J Aust 2012;196(1):50-53. doi: 10.5694/mja11.10698.

3. Chen EYH, Bell JS, llomaki J, et al. Medication regimen complexity in 8 Australian residential aged care facilities: Impact of age, length of stay, comorbidity, frailty, and dependence in activities of daily living. Clin Interv Aging 2019;14:1783-95. doi: 10.2147/CIA.S216705

4. Mc Namara KP, Breken BD, Alzubaidi HT, et al. Health professional perspectives on the management of multimorbidity and polypharmacy for older patients in Australia. Age Ageing 2017;46(2):291-99. doi: 10.1093/ageing/afw200.

5. Lindquist LE, Lindquist LM, Zichuhr L, Fiesma E, Wolf MS. Unnecessary complexity of home medication regimens among seniors. Patient Educ Couns 2014;96(1):93-97. doi: 10.1016/j. pec.2014.03.022

6. Wolf MS, Curtis LM, Waite K, et al. Helping patients simplify and safely use complex prescription regimens. Arch Intern Med 2011;171(4):300-05. doi: 10.1001/ archinternmed.2011.39.

7. Pitkälä KH, Suominen MH, Bell JS, Strandberg TE. Herbal medications and other dietary supplements. A clinical review for physicians caring for older people. Ann Med 2016;48(8):586-602. doi: 10.1080/07853890.2016.1197414.

8. Wimmer BC, Cross AJ, Jokanovic N, et al. Clinica outcomes associated with medication regimen complexity in older people: A systematic review. J Am Geriatr Soc 2017;65(4):747-53. doi: 10.1111/ jgs.14682.

9. Wimmer BC, Dent E, Visvanathan R, et al. Polypharmacy and medication regimen complexity as factors associated with hospital discharge destination among older people: A prospective cohort study. Drugs Aging 2014;31(8):623-30. doi: 10.1007/s40266-014-0185-1.

10. Coleman Cl, Limone B, Sobieraj DM, et al. Dosing frequency and medication adherence in chronic disease. J Manage Care Pharm 2012;18(7):527-39. doi: 10.18553/jmcp.2012.18.7.527.

11. Richter A, Anton SF, Koch P, Dennett SL. The impact of reducing dose frequency on health outcomes. Clin Ther 2003:25(8):2307-35:discussion 2306 doi: 10.1016/s0149-2918(03)80222-9.

12. Onder G, Vetrano DL, Marengoni $A$, et al. Accounting for frailty when treating chronic diseases. Eur J Intern Med 2018;56:49-52. doi: 10.1016/j.ejim.2018.02.021.

13. Grant JS, Graven LJ. Problems experienced by informal caregivers of individuals with heart failure: An integrative review. Int J Nurs Stud 2018;80:41-46. doi: 10.1016/j.ijnurstu.2017.12.016.

14. Thomson MS, Gruneir A, Lee M, et al. Nursing time devoted to medication administration in long-term care: Clinical, safety, and resource implications. J Am Geriatr Soc 2009;57(2):266-72. doi: 10.1111/j.1532-5415.2008.02101.x.

15. Sluggett JK, Chen EYH, llomäki J, et al. Reducing the burden of complex medication regimens: SImplification of Medications Prescribed to Long-tErm care Residents (SIMPLER) cluster randomized controlled trial. J Am Med Dir Assoc 2020;21(8):1114-20.e4. doi: 10.1016/j. jamda.2020.02.003. 
16. Chen EYH, Sluggett JK, llomäki J, et al.

Development and validation of the Medication Regimen Simplification Guide for Residential Aged CarE (MRS GRACE). Clin Interv Aging 2018;13:975-86. doi: 10.2147/CIA.S158417.

17. Turner JP, Edwards S, Stanners M, Shakib S, Bell JS. What factors are important for deprescribing in Australian long-term care facilities? Perspectives of residents and health professionals. BMJ Open 2016;6(3):e009781. doi: 10.1136/bmjopen-2015-009781.

18. Australian Commission on Safety and Quality in Health Care. Medication reconciliation. Sydney, NSW: ACSQHC, 2019. Available at www. safetyandquality.gov.au/our-work/medicationsafety/medication-reconciliation [Accessed 10 July 2020].

19. The Royal Australian College of General Practitioners. RACGP aged care clinical guide (Silver Book). 5th edn. East Melbourne, Vic: RACGP, 2019.

20. Chen EYH, Wang KN, Sluggett JK, et al. Process, impact and outcomes of medication review in Australian residential aged care facilities: A systematic review. Australas J Ageing 2019;38 Suppl 2:9-25. doi: 10.1111/ajag.12676.

21. Jokanovic N, Tan ECK, van den Bosch D, Kirkpatrick CM, Dooley MJ, Bell JS. Clinical medication review in Australia: A systematic review. Res Social Adm Pharm 2016;12(3):384-418. doi: 10.1016/j.sapharm.2015.06.007.

22. Anderson K, Stowasser D, Freeman C, Scott I. Prescriber barriers and enablers to minimising potentially inappropriate medications in adults: A systematic review and thematic synthesis. BM Open 2014;4:e006544. doi: 10.1136/bmjopen2014-006544.

23. Thompson W, Farrell B, Welch V, et al. Continuation or deprescribing of proton pump inhibitors: A consult patient decision aid. Can Pharm J (Ott) 2019;152(1):18-22. doi: 10.1177/1715163518816719.

24. Stacey D, Légaré $F$, Lewis $K$, et al. Decision aids for people facing health treatment or screening decisions. Cochrane Database Syst Rev 2014;4(4):CD001431. doi: 10.1002/14651858. CD001431.pub5.

25. Kalogianis MJ, Wimmer BC, Turner JP, et al. Are residents of aged care facilities willing to have their medications deprescribed? Res Social Adm Pharm 2016;12(5):784-88. doi: 10.1016/j. sapharm.2015.12.004.

26. Sluggett JK, Hopkins RE, Chen EYH, et al. Impact of medication regimen simplification on medication administration times and health outcomes in residential aged care: 12 month follow up of the SIMPLER randomized controlled trial. J Clin Med 2020;9(4):1053. doi: 10.3390/ jcm9041053.

27. Sluggett JK, Ooi CE, Gibson S, et al. Simplifying medication regimens for people receiving community-based home care services: Outcomes of a non-randomized pilot and feasibility study. Clin Interv Aging 2020;15:797. doi: 10.2147/CIA S248377.

28. Elliott RA. Appropriate use of dose administration aids. Aust Prescr 2014;37(2):46-50. doi: 10.18773/ austprescr.2014.020.

29. Australian Commission on Safety and Quality in Health Care. NSQHS Standards. Action 4.12 - Provision of a medicines list. Sydney, NSW: ACSQHC, 2019. Available at www. safetyandquality.gov.au/standards/nsqhsstandards/medication-safety-standard/ continuity-medication-management/action-412 [Accessed 10 July 2020]. 